\title{
Asymmetric Perturbation of Carboxyl Chromophores in Optically Active Random Copolymers of Acrylic Acid and (+) $\mathrm{N}$-s-Butyl- $N$-methylacrylamide
}

\author{
Christian BRAUD and Michel VerT \\ Laboratoire de Chimie Macromoléculaire-ERA CNRS \\ 471-, Faculté des Sciences et des Techniques, \\ Univerité de Haute-Normandie, 76130 \\ Mont-Saint-Aignan, France.
}

(Received March 26, 1980)

\begin{abstract}
Investigations were made of ultraviolet, optical rotatory dispersion, and circular dichroism of acrylic acid $-(+) N$-s-butyl- $N$-methylacrylamide random copolymers of different composition (COP Y, with $Y=$ percentage of achiral acid repeat units). The nonlinear compositiondependence of optical rotatory dispersion and circular dichroism spectra is discussed with regard to the properties of homopolymers corresponding to amide and acid repeating units, i.e., $(-)$ poly[ $N$ $s$-butyl- $N$-methylacrylamide] and poly(acrylic acid), respectively. It is shown that some of the achiral acid units were asymmetrically perturbed because of a neighboring effect of chiral amide units. This finding well accounts for the nonlinear-composition-dependent chiroptical properties of COP $Y$, in spite of their random-coil structure in solution.
\end{abstract}

KEY WORDS Optically Active Copolymers / Chromophores / Ultraviolet Spectra / Optical Rotatory Dispersion / Circular Dichroism / Induced Optical Activity / Random-Coil Conformation /

The optical activity of copolymers of two chiral repeating units or of achiral and chiral units raises particular problems not found in the optical activity of homopolymers. For randomly coiled copolymers, a variation in optical activity in proportion to composition is generally expected, especially for macromolecules derived from an optically-active monomer with an achiral mono-substituted comonomer. Linear relationships were effectively found for various optically-active copolymers whose chains were obviously randomly coiled in solution. ${ }^{1-4}$ However, deviations from linearity were reported for more or less stereoregular copolymers. These deviations are generally interpreted in term of ordered macromolecular conformations, especially in the cases of stereoregular $\alpha$-olefins or vinylic copolymers of achiral and chiral repeat units in which achiral units were found optically-active because of helical structures having a preferential screw sense brought about by chiral units. $^{5-8}$

In a previous paper, we have reported that optical rotatory power of random copolymers of acrylic acid and (+) and/or (-) $N$-s-butyl- $N$-methylacrylamides (COP Y, with $Y=100 \mathrm{~m} / \mathrm{n}=$ percentage of achiral acid units in copolymer chains), as obtained by progressive acidic hydrolysis of parent radically-polymerized homopolyacrylamides (PAAm), does not vary linearly with the content in chiral amide repeating units. ${ }^{9}$
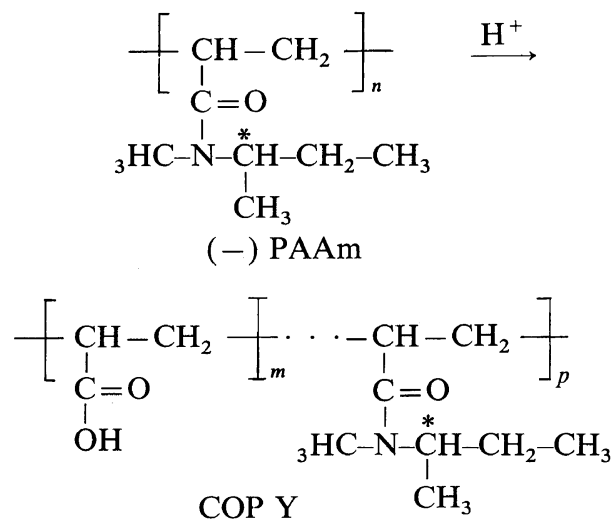
The possibility for stereoregularity and for stereoordered conformations to be responsible for this nonlinear dependence has already been discussed. For various reasons (including the synthesis of parent homopolyacrylamides by radical polymerization, ${ }^{10}$ the first-order kinetics of the homopolyacrylamide acidic hydrolysis, which yields the copolymers, and the linear variation of the optical activity of PAAm stereocopolymers with the enantiomeric composition ${ }^{9}$ ), it has been concluded that COP Y macromolecules are randomly coiled in solution and that acid and amide repeating units are randomly distributed in polymer chains. ${ }^{9}$

To account for the nonlinear variation of optical rotatory power with composition in the absence of ordered conformations, it has been suggested that chiroptical parameters, considered as specific of both types of repeating units, are actually composition-dependent because of modifying effects due to the chemical nature of changing immediate neighbors. Accordingly, optical activity of COP Y's has been considered to result from the partial contributions of eight independent opticallyactive species defined as the central units of various triads found in a random copolymer of $a$ and $b$ units, i.e., triads a $a \underline{a} a, a \underline{a} b, b \underline{a} a, b \underline{a} b, a b a, b \underline{b} a, a \underline{b} b$, and $b \underline{b} b$. It is significant to note that $a \underline{a} b=b a$ a and $\mathrm{b} \underline{b} a=a \underline{b} b$, in so far as acrylic type main chains are concerned.

On the basis of a bernouillian distribution of a and $b$ units in COP Y chains, eq 1 was derived and was found to fit the experimental data very well, ${ }^{9}$

$$
\begin{aligned}
\langle m\rangle & =(1-Y / 100)^{3}[\Phi]_{\mathrm{a \underline {a } a}}+(Y / 100)^{3}[\Phi]_{\mathrm{b} \underline{\mathrm{b}} \mathrm{b}} \\
& +Y / 100(1-Y / 100)\left(\delta Y / 100+\delta^{\prime}\right)
\end{aligned}
$$

with

$$
\delta=2[\Phi]_{\text {abb }}-2[\Phi]_{\text {aạb }}+[\Phi]_{\text {bạ }}-[\Phi]_{\text {aba }}
$$

and

$$
\delta^{\prime}=2[\Phi]_{\text {aa } b}+[\Phi]_{\text {a ba }}
$$

In eq $1,\langle m\rangle$ and $[\Phi]_{\mathrm{iji}}$ figure the mean-residue optical rotatory power of COP Y's and the molar rotatory power of the various optically-active species involved, respectively. $\delta$ and $\delta^{\prime}$ are deduced from slopes of the $\langle m\rangle=f(Y)$ experimental curve for $Y=0$ :

$$
\left((\partial\langle m\rangle / \partial Y)_{Y=0}=\left(\delta^{\prime}-3[\Phi]_{\mathrm{aa} a} \mathrm{a}\right) / 100\right),
$$

and for $Y=100$ :

$$
\left((\partial\langle m\rangle / \partial Y)_{Y=100}=\left(3[\Phi]_{\mathrm{bbb}}-\delta-\delta^{\prime}\right) / 100\right)
$$

It should be noted that eq 1 can be expressed for the mean-residue absorption coefficient and also for the mean-residue elipticity, considering the $\varepsilon_{\mathrm{iii}}$ 's and $[\theta]_{\mathrm{iii}}$ 's.

So far, it has not been possible to calculate the specific contributions $[\Phi]_{\mathrm{iji}}$ of the various opticallyactive species, because of the lack of data regarding the copolymers with nonrandom distribution of a and $b$ units required for having a sufficient number of independent relationships to solve eq $1 .{ }^{9}$ The direct evidence for the optical activity of ibi species, and particularly of $\mathrm{COOH}$ chromophores is not possible either, because of the overlapping of $n \rightarrow \pi^{*}$ and $\pi \rightarrow \pi^{*}$ cotton effects of the amide chromophores with the $n \rightarrow \pi^{*}$ electronic transition of $\mathrm{COOH}$ chromophores, the latter transition being usually located around $210 \mathrm{~nm} .{ }^{11}$

In this paper, we wish to report our attempts to demonstrate the optical activity of ibi species, and particularly that of the $n \rightarrow \pi^{*}$ electronic transition of $\mathrm{COOH}$ chromophores. Ultraviolet (UV), optical rotatory dispersion (ORD), and circular dichroism (CD) spectra of various COP Y are described. A comparison with data obtained for (-) PAAm and for poly(acrylic acid), which are the homopolymers corresponding to the units present in COP Y chains, shows that there are effects from the copolymer composition on these spectra. The need for optically-active $\mathrm{COOH}$ chromophores to account for experimental data is discussed.

\section{EXPERIMENTAL}

\section{The Synthesis of Various COP Y Copolymers}

COP Y copolymers considered herein were obtained by the partial hydrolysis of (-) PAAm (homopolyacrylamide) as previously reported. ${ }^{9-10}$ Two different samples of $(-)$ PAAm $((-)$ PAAm I and (-) PAAm II) having different molecular weights were used as parent compounds. For both samples, the low-molecular-weight fractions were separated by coacervative precipitation using methanol-water as the solvent-nonsolvent system. The intrinsic viscosity and optical rotatory power for the different COP Y thus obtained are given in Table I.

\section{Measurements}

UV absorption. UV spectra were recorded at 
Chromophores in Optically Active Copolymers

Table I. Physico-chemical characteristics of COP Y copolymers and of parent (-) PAAm

\begin{tabular}{|c|c|c|c|c|c|}
\hline \multirow{2}{*}{ COP Y } & $\mathrm{Y}^{\mathrm{a}}$ & \multirow{2}{*}{$\begin{array}{c}\text { Parent } \\
(-) \text { PAAm }\end{array}$} & \multirow{2}{*}{$M_{\mathrm{R}}$} & {$[\eta]^{\mathbf{b}}$} & $\langle m\rangle^{\mathrm{c}}$ \\
\hline & $\%$ & & & $100 \mathrm{~cm}^{3} \mathrm{~g}^{-1}$ & $\operatorname{deg} \mathrm{cm}^{-2} \mathrm{dmol}^{-1}$ \\
\hline COP 0 & - & I & 141 & 0.41 & -73.5 \\
\hline " & - & II & "' & 0.34 & -73.1 \\
\hline COP 19 & 19.0 & I & 127.9 & 0.35 & -34.0 \\
\hline COP 25 & 24.6 & II & 131.9 & 0.27 & -26.4 \\
\hline COP 37 & 36.8 & I & 115.6 & 0.29 & -9.5 \\
\hline COP 39 & 38.9 & II & 114.1 & 0.24 & -6.5 \\
\hline COP 52 & 52.1 & I & 105.0 & 0.27 & +4.0 \\
\hline COP 62 & 62.8 & II & 98.4 & - & +8.2 \\
\hline COP 72 & 72.0 & I & 91.3 & 0.32 & +10.0 \\
\hline COP 90 & 90.5 & II & 78.6 & 0.36 & +4.7 \\
\hline COP 100 & 100.0 & I & 72 & - & 0.0 \\
\hline
\end{tabular}

a As determined by non-aqueous titration of $-\mathrm{COOH}$ groups. ${ }^{9}$

b In methanol at $15.0^{\circ} \mathrm{C}$.

c In methanol, $c=(0.5-1.0) \mathrm{g} 100 \mathrm{~cm}^{-3}$.

room temperature by using a CARY-15 spectrophotometer whose monochromator and cell compartment were flushed with nitrogen.

$O R D$. ORD spectra were recorded with a "Spectropol I" FICA spectropolarimeter thermostated at $25.0^{\circ} \mathrm{C}$.

$C D$. CD spectra were obtained at room temperature by using a JASCO J-40-B dichrometer. Data at given wavelengths were obtained by setting the monochromator in the proper position in order to avoid inaccuracies of data deduced from spectra directly.

UV, ORD, and CD data are expressed in terms of the mean residue and thus, are referred to the meanresidue molecular weight,

$$
M_{\mathrm{r}}=(72 Y / 100+141(100-Y) / 100)
$$

\section{RESULTS AND DISCUSSION}

Investigations were made of the UV spectra of the parent (-) PAAm and of the resulting COP Y copolymers (including COP 100 which corresponds to poly(acrylic acid)) in methanol, a solvent used for all the copolymers. Figure la shows the UV spectra of (-) PAAm (COP 0) and of COP 100. Figure 1b represents the variations of the mean-residue extinction coefficient of $\mathrm{COP} Y$ with regard to the composition for three different wavelengths: $\lambda=200 \mathrm{~nm}$, the wavelength corresponding to the

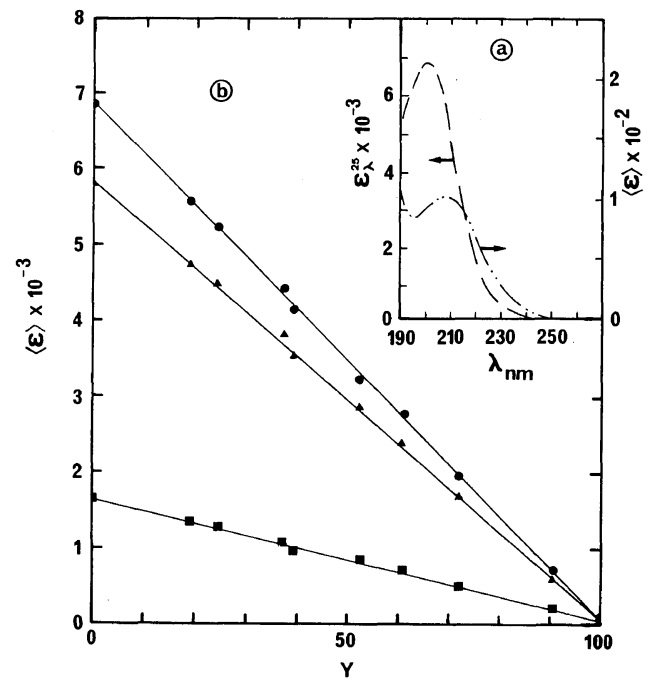

Figure 1. (a) UV absorption spectra of (-) PAAm $(--)$ and of COP $100(-\cdots-\cdots)$ in methanol.

(b) Composition-dependence of mean-residue extinction coefficient of COP Y in methanol at three wavelengths:

- $\lambda=200 \mathrm{~nm} ; \boldsymbol{\Delta}, \lambda=207 \mathrm{~nm} ; \boldsymbol{\square}, \lambda=220 \mathrm{~nm}$.

maximum observed in the (-) PAAm spectrum; $\lambda=207 \mathrm{~nm}$, which corresponds to the maximum of the COP 100 spectrum, and $\lambda=220 \mathrm{~nm}$, a wavelength which corresponds to high-wavelength hedges of spectra of both homopolymers. In the range $190-250 \mathrm{~nm},(-)$ PAAm absorbed much more than COP 100. Though the strong absorption band 
centered at $200 \mathrm{~nm}\left(\varepsilon_{200}=6930\right)$ seemed simple, it actually resulted from several electronic transitions including the $n \rightarrow \pi^{*}$ and the $\pi \rightarrow \pi^{*}$ transitions as already shown by comparison with CD spectra. ${ }^{12}$ In contrast, the weak band observed at $207 \mathrm{~nm}$ for COP 100 corresponds to the $n \rightarrow \pi^{*}$ electronic transition of the $\mathrm{COOH}$ chromophore while the stronger $\pi \rightarrow \pi^{*}$ is located below $190 \mathrm{~nm}$, which is the limit imposed by the solvent absorption. The linear variation of $\varepsilon$ with $Y$, regardless of the wavelength, indicated that the UV absorption of COP Y copolymers depends on two well-defined absorbing species only, i.e., the amide repeating units and the acid units behaving as in homopolymers.

The ORD spectra of COP Y copolymers in methanol are shown in Figure 2. Since the same solvent was used for all the compounds, the correction due to the Lorentz factor was not determined. The curve corresponding to (-) PAAm was negative and normal according to Platt's definitions. ${ }^{13}$ As the proportion of the acid units increased, ORD became more complex. Instead of tending asymptotically to the zero axis as $Y$ increased, the optical rotations observed in the highwavelength region became positive and the cross-

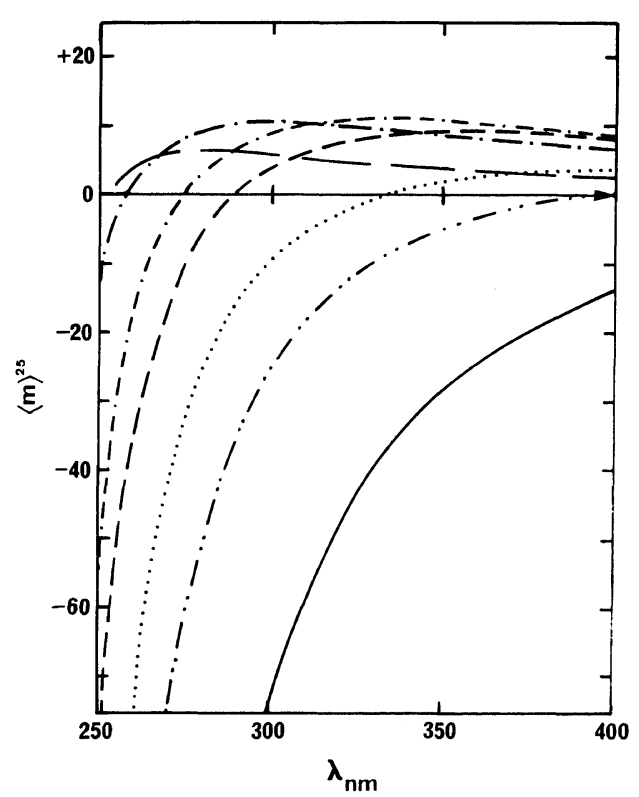

Figure 2. ORD spectra of COP Y in methanol $(c=0.5$

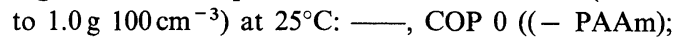
$-\cdots-\cdots, \operatorname{COP} 25 ; \cdots \cdots$, COP 37 ; ----, COP 52 ; --.-.-, COP $62 ;-\cdot-$, COP $72 ;--$, COP 90. over point $(\langle m\rangle=0)$ was blue-shifted. These features, together with the nonlinear variation of $\langle m\rangle_{300}$ with composition already discussed, ${ }^{9}$ show that COP Y copolymers have a particular behavior, compared to (-) PAAm and COP 100 homopolymers, in regard to optical activity.

The CD spectra of COP Y in methanol are shown in Figure 3. Though magnitudes differed considerably because of the variation of the content in the chiral acrylamide repeat units, the shapes of the various spectra were similar. These spectra exhibited two CD bands opposite in signs and approximately of the same magnitudes, and whose maxima were located at about $197 \mathrm{~nm}$ (positive band) and about $215 \mathrm{~nm}$ (negative band). A spectrum having the same features was found for the parent homopolyacrylamide, ${ }^{12}$ as shown in Figure 3. In this case, the possibility for exciton coupling between amide chromophores to account for the bisignate $C D$ curve observed in the range of the amide $n \rightarrow \pi^{*}$ electronic transition was precluded, and the particular shape of the CD spectrum has been considered to be attributable to the behavior of noncoupled $N$ $N$-disubstituted amide chromophores. ${ }^{12}$ The CD spectra of COP Y copolymers reported in this paper

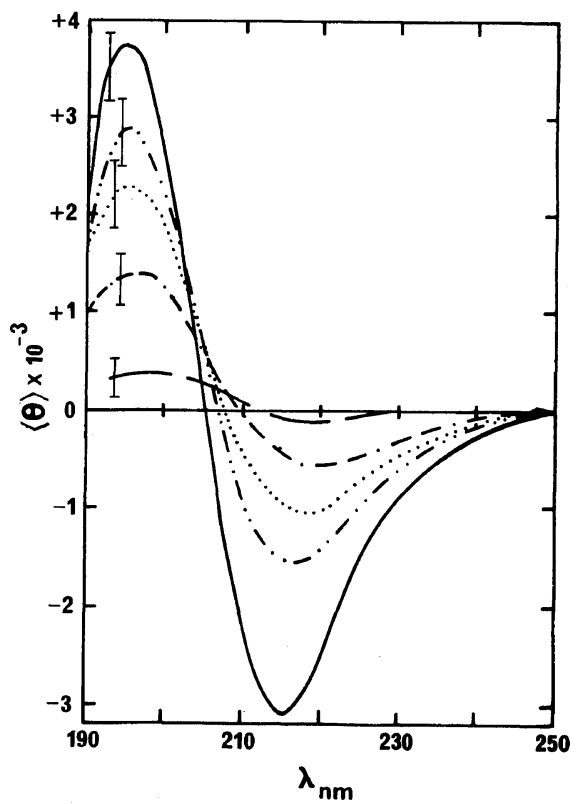

Figure 3. $\mathrm{CD}$ spectra of COP $\mathrm{Y}$ in methanol $(c=0.1$ to

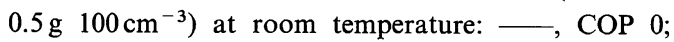
$-\cdots-\cdots$, COP $25 ; \cdots \cdots$, COP $39 ;--\cdot--$, COP 62 ; - COP 90 . 
can be accounted for in this manner too. Indeed, exciton coupling between amide chromophores, if present in partially hydrolyzed copolymers, should depend on the distance separating interacting groups and, thus, on the content in acid repeating units. This is obviously not the case since copolymers with a low content in chiral acrylamide units still show two $\mathrm{CD}$ bands opposite in signs. On the other hand, it is remarkable that the cross-over point observed in $\mathrm{CD}$ spectra depends on composition. The higher the content of macromolecules in acidic units, the higher the wavelength of the cross-over point. This feature shows that the spectra of COP Y copolymers do not result from a linear combination of spectra of corresponding homopolymers, i.e., they do not vary proportionally to the content in chiral amide units. However, this statement is not valid for all the wavelengths, as shown in Figure 4 which gives the compositiondependence of the mean-residue ellipticity of the copolymers for different wavelenghts: 195, 210, and $215 \mathrm{~nm}$. Indeed, bended curves were obtained for 210 and $215 \mathrm{~nm}$, but for $195 \mathrm{~nm}$, the curve may be considered as a straight line, in spite of the large uncertainties in data arising from the low signal/noise ratio. Therefore, it is concluded that $\mathrm{CD}$ modifications due to immediate neighbors are limited to the spectral zone corresponding to the high-wavelength band, in so far as accessible parts of CD spectra are concerned. Such modifications can affect either the amide unit chromophores

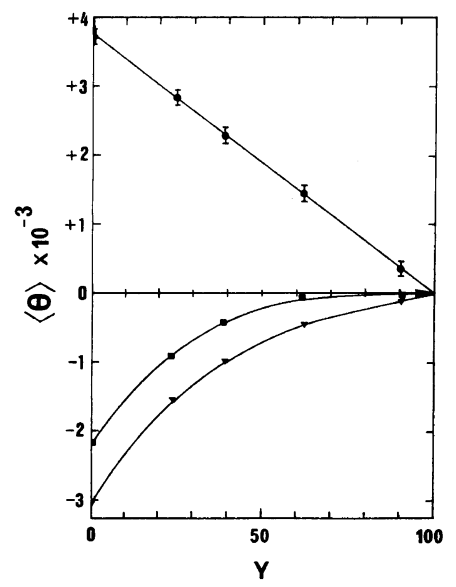

Figure 4. Composition-dependence of mean-residue ellipticity of COP $\mathrm{Y}$ in methanol $(c=0.1$ to $0.5 \mathrm{~g}$ $100 \mathrm{~cm}^{-3}$ ) for three wavelengths: $O, \lambda=195 \mathrm{~nm}$; $\lambda=210 \mathrm{~nm} ; \boldsymbol{\Delta}, \lambda=215 \mathrm{~nm}$. (variations in magnitude and/or position of cotton effects) or acid unit chromophores (extra cotton effects due to induced optical activity in corresponding electronic transitions). However, it is more likely that both of these are related because of reciprocal effects, as suggested in eq 1 .

Figure 4 shows that $C D$ experimental data well agree with COP Y mean-residue ellipticity calculated using eq 1 , written in terms of ellipticity, using the values of $[\theta]_{\mathrm{aa} a \mathrm{a}},[\theta]_{\mathrm{bbb}}, \delta$, and $\delta^{\prime}$ listed in Table II. However, this agreement does not allow us to know whether the ibi species actually contribute to $\mathrm{CD}$ at $215 \mathrm{~nm}$, since the values of the different $[\theta]_{i i i}$ cannot be calculated because of the lack of independent relationships.

To overcome this problem, an indirect method is used in which it is shown that the assumption $[X]_{\mathrm{ibi}}=0(X=[\Phi]$ or $[\theta])$ i.e., optical inactivity for acid repeat units, leads to inconsistent evaluations of partial ORD and CD contributions of the iai species. It is assumed that only chiral amide repeating units are optically active in COP Y macromolecules. In this case, eq 1 is simplified and partial contributions of iai species can be deduced from $\delta$ and $\delta^{\prime}$ through eq 2 and 3 where $[X]_{\mathrm{aaa}}$ is considered identical to the (-) PAAm mean residue ellipticity, since all repeating units of this homopolymer are of the aa type.

$$
\begin{aligned}
& {[X]_{\mathrm{b} \underline{\mathrm{a}} \mathrm{b}}=\delta+\delta^{\prime}} \\
& {[X]_{\mathrm{a} \underline{\mathrm{a}} \mathrm{b}}=\delta^{\prime} / 2}
\end{aligned}
$$

Figure 5 represents $\mathrm{CD}$ partial contributions of species iai thus obtained from the $\langle\theta\rangle=f(Y)$ curves corresponding to various wavelengths, as exemplified in Figure 4 for 190, 200, and $215 \mathrm{~nm}$.

Partial ORD contributions of species iai were evaluated similarly (Figure 6), from the $\langle m\rangle=f(Y)$

Table II. Experimental values of parameters $[\theta]_{\mathbf{a a a}},[\theta]_{\mathbf{b b b}}, \delta$, and $\delta^{\prime}$ used for calculated $\langle\theta\rangle=f(Y)$ curves shown in Figure 4

\begin{tabular}{ccccc}
\hline$\lambda$ & & & & \\
\cline { 1 - 1 } $\mathrm{nm}$ & {$[\theta]_{\mathbf{a} \underline{\mathbf{a}}}{ }^{\mathbf{a}}$} & {$[\theta]_{\mathbf{b} \underline{\mathbf{b}}{ }^{\mathbf{a}}}$} & $\delta$ & $\delta^{\prime}$ \\
\hline 195 & +3730 & 0 & -3730 & +7460 \\
210 & -2200 & 0 & -800 & +600 \\
215 & -3080 & 0 & +100 & -1400 \\
\hline
\end{tabular}

a a, amide repeating units; $b$, acid ones. 
C. Braud and M. VerT

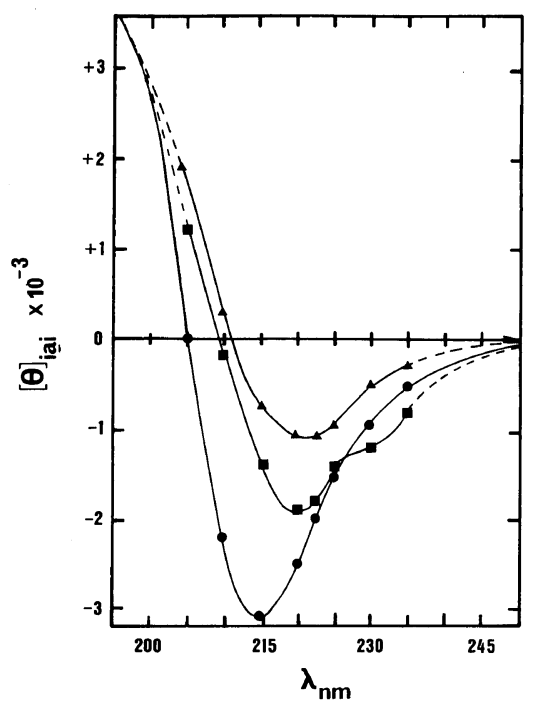

Figure 5. Partial $C D$ contributions of species aaa $(\boldsymbol{O})$, aab = baa $(\boldsymbol{\Lambda})$, and bab $(\boldsymbol{\square})$, as deduced from experimental CD spectra of COP Y copolymers by the use of eq 1 assuming that ibi species are not optically active $\left([\theta]_{\underline{b} \underline{b}}=[\theta]_{a \underline{b} b}=[\theta]_{\underline{b} \underline{a}}=[\theta]_{a \underline{a b}}=0\right)$.

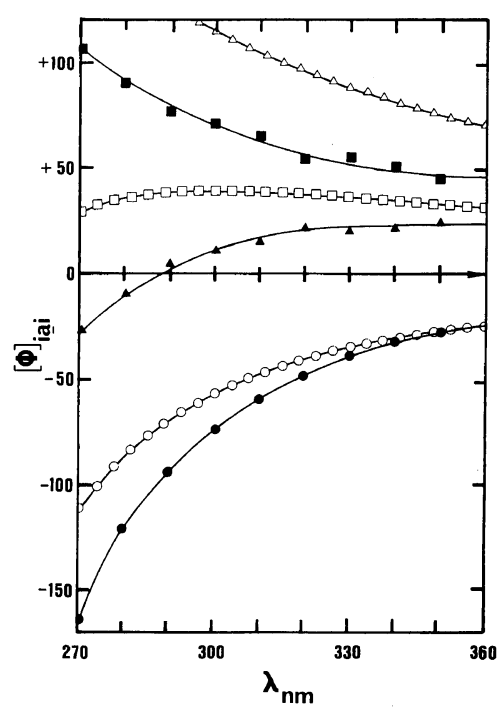

Figure 6. Partial monomolar ORD contributions of species aa a $(O)$, a $\underline{a} b=$ baa $(\triangle)$, and bab $(\square)$ : as deduced from ORD spectra shown in Figure 2 by using eq 1,2 , and 3 (full symbols), and also a calculated by KronigKramers transforms applied to CD spectra of Figure 5 resolved in three Gaussian cotton effects whose characteristics are listed in Table III (open symbols). curves deduced from ORD spectra shown in Figure 2. If the assumption that ibi species are optically inactive is right, the partial ORD and partial CD curves for each iai species can be related through Kronig-Kramers transforms. ${ }^{14}$ Therefore, partial ORD contributions were also calculated (Figure 6) by using eq 4, derived from Kronig-Kramers transforms, ${ }^{14}$ and which was applied to iai CD spectra in Figure 5 resolved in three Gaussian cotton effects whose characteristics are given in Table III.

$[\Phi]=\sum_{j=1}^{n} \frac{R_{j}}{0.696 \times 10^{-42}} \frac{2}{\pi} \frac{\lambda_{j}^{2}}{\lambda^{2}-\lambda_{j}^{2}} \quad$ with $\left.\left.\lambda\right\rangle \gg\right\rangle \lambda_{j}$

and with

$$
R_{j}=0.696 \times 10^{-42} \sqrt{\pi}[\theta]_{j}^{\circ} \Delta \lambda_{j} / \lambda_{j}
$$

in cgs units

In this equation, $[\theta]_{j}^{\circ}$ is the molar ellipticity at the wavelength $\lambda_{j}$ characterizing the electronic transition $j$, and $\Delta \lambda_{j}$ is the band half-width at $[\theta]_{j}=1 / e[\theta]_{j}^{\circ}$. For carrying out the calculation, the number of cotton effects was selected as three with regard to previous calculations carried out for (-) PAAm. ${ }^{12}$

Both evaluations of ORD partial contributions of species aaa were in fairly good agreement. But, for aab (baa a) and bạb species, there were drastic

Table III. Characteristics of partial Gaussian cotton effects contributing to $\mathrm{CD}$ spectra of species aaa , baa $\equiv \mathbf{a} \underline{a} b$, and bab, as deduced from calculated spectra shown in Figure 5

\begin{tabular}{|c|c|c|c|c|}
\hline \multirow{2}{*}{ Species } & $\lambda_{j}$ & $\Delta \lambda_{j}$ & {$[\theta]_{j}^{0}$} & $R_{j} \times 10^{42 \mathrm{a}}$ \\
\hline & $\mathrm{nm}$ & $\mathrm{nm}$ & $\operatorname{deg} \mathrm{cm}^{-2} \mathrm{dmol}^{-1}$ & cgs \\
\hline \multirow{3}{*}{$a \underline{a} a^{b}$} & 225 & 8 & +450 & +20 \\
\hline & 209 & 19 & -4150 & -465 \\
\hline & 197 & 12 & +6150 & +462 \\
\hline \multirow{3}{*}{ bạa } & 224 & 8 & -200 & -9 \\
\hline & 206 & 17 & -4500 & -458 \\
\hline & 199 & 16 & +7000 & +694 \\
\hline \multirow{3}{*}{ bạb } & 224 & 7 & +350 & +13 \\
\hline & 209 & 20 & -4200 & -496 \\
\hline & 198 & 16 & +6400 & +638 \\
\hline
\end{tabular}

a Calculated by using eq 4.

b Values corresponding to (-) PAAm in methanol from ref 12 . 
discrepancies. Bends of ORD contributions calculated according to both ways were opposite. Furthermore, a comparison of both sets of calculated data shows that two different series, aạa $<$ a a $b<$ b ab and aa $a$ when going from negative to positive rotations. From these inconsistencies, it is concluded that the assumption $[X]_{i \underline{i} i}=0$ is meaningless and, thus, the acid species $i \underline{b} i$ are more or less optically active in COP Y copolymers, depending on the nature of the neighbors $i$.

Among the various electronic transitions due to chromophores present in acid repeat units $(-\mathrm{C}-\mathrm{C}-$, $-\mathrm{C}-\mathrm{H}$ and $\mathrm{COOH}$ ); the $n \rightarrow \pi^{*}$ transition of $\mathrm{COOH}$ chromophores is the only one which can be directly observed in the $185-250 \mathrm{~nm}$ spectral range. However, it is of value to recall that $-\mathrm{C}-\mathrm{C}$ - and $-\mathrm{C}-\mathrm{H}$ electronic transitions located below $185 \mathrm{~nm}$, in a zone inaccessible to ordinary CD meters, can contribute to ORD up to the visible spectral range because of the dispersive effect. This is the case for optically-active saturated aliphatic hydrocarbons, as in example. ${ }^{15}$

In order to localize the cotton effect due to the $n \rightarrow \pi^{*}$ electronic transition of $\mathrm{COOH}$ chromophores in COP Y CD spectra, (+) $S$-2-methylbutanoic acid was taken as a suitable model compound. Figure 7 shows the CD spectrum of this model compound in acetonitrile. In the $185-250 \mathrm{~nm}$ spectral range, a positive CD band, centered at $217-218 \mathrm{~nm}$, was observed. It is of a particular interest to note that this feature is common to many carboxylic acids

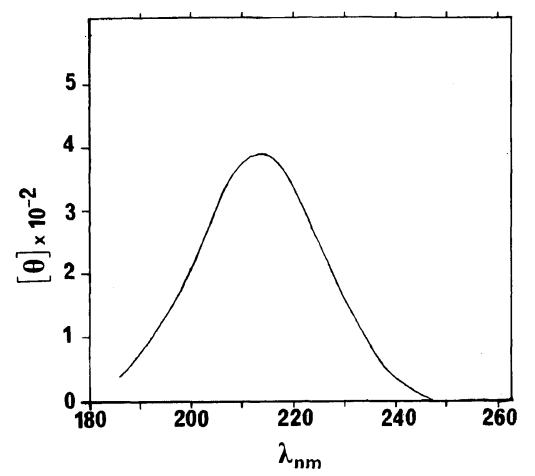

Figure 7. CD spectrum of (+) $S$-2-methylbutanoic acid taken as a suitable model of COP Y acid repeating units asymmetrically perturbed by chiral centers of amide immediate neighbors $\left(c=1.0 \mathrm{~g} 100 \mathrm{~cm}^{-3}\right.$ in acetonitrile). such as 2-methyl pentanoic acid ${ }^{16}$ or polyacids such as maleic acid/chiral alkyl vinyl ethers alternating copolymers. ${ }^{17}$ This point constitutes further evidence for the optical activity of some of the $\mathrm{COOH}$ chromophores in COP Y. Indeed, the linear $\langle\theta\rangle=f(Y)$ curve observed at $190 \mathrm{~nm}$, and nonlinear curves at 210 and $215 \mathrm{~nm}$ (Figure 4), can be readily accounted for on the basis of the presence of $\mathrm{COOH}$ $n \rightarrow \pi^{*}$ cotton effects since the CD contribution of this type of cotton effect is negligible at $190 \mathrm{~nm}$ and maximum in the $215 \mathrm{~nm}$ zone.

Finally, our work points to the fact that even randomly-coiled copolymers of chiral and achiral monomers can show extra cotton effects associated with electronic transitions of the latter, and to nonlinear variations of optical activity with composition. It is likely that spacial locations of chiral centers, and chromophores, are critical factors for determining neighboring effects, especially for magnitude of the extra cotton effects. One must keep in mind that, in polymers, actual distances can be much shorter than those given by bond lengths, because of foldings which may be significant in poor solvents for example. The proposed neighboring effect of vicinal units well accounts for the fact that specific rotations of the copolymers of $S-4$ methyl-2- $N, N$-dimethylaminopentyl methacrylate with styrene are not proportional to the content of the chiral units as recently reported by Sato et al ${ }^{18}$

A rigorous treatment of the problems posed by the chiroptical properties of optically-active copolymers of chiral and achiral units requires a series of compounds obtained by different routes with known random and nonrandom distribution of counits. Data corresponding to such a series of optically active copolymers does not exist in the literature. The COP Y series was synthesized especially for this purpose but, so far, it has not been possible to determined the co-unit distribution by ${ }^{1} \mathrm{H}$ and ${ }^{13} \mathrm{C}$ NMR because of the lack of suitable fine structures in the NMR peaks. Determination of triad statistics of co-units by NMR was shown to be possible for a series of nonoptically active copolymers obtained by partial hydrolysis of syndiotactic poly(methyl methacrylate) ${ }^{19}$ It would be of interest to consider, in the future, a similar series of stereoregular copolymers with chiral substituents in the side-chains. 


\section{REFERENCES}

1. K. Matsuzaki and T. Sugimoto, Makromol. Chem., 164, 127 (1973).

2. R. C. Schulz and E. Kaiser, Makromol. Chem., 86, 80 (1965).

3. H. Yamaguchi, H. Ueno, and Y. Minoura, J. Polym. Sci., A-1, 9, 897 (1971).

4. H. Yamaguchi and Y. Minoura, J. Polym. Sci., A-1, 8, 1467 (1970).

5. C. Carlini, F. Ciardelli, and P. Pino, Makromol. Chem., 119, 244 (1968).

6. H. Yuki, K. Ohta, and N. Yajima, Polym. J., 1, 164 (1970).

7. K. Ohta, K. Hatada, Y. Okamoto, and H. Yuki, $J$. Polym. Sci., Polym. Lett. Ed., 16, 545 (1978).

8. N. Majumbar, C. Carlini, R. Nocci, F. Ciardelli, and R. C. Schulz, Makromol. Chem., 177, 3619 (1976).

9. C. Braud and M. Vert, Polymer, 16, 115 (1975).

10. C. Braud, M. Vert, and E. Selegny, Makromol. Chem., 175, 775 (1974).
11. H. H. Jaffe and M. Orchin, "Theory and Applications of Ultraviolet Spectroscopy," Wiley \& Sons, New York, N.Y., 1962, p 180.

12. C. Braud and M. Vert, Isr. J. Chem., 15, 39 (1976/77).

13. J. R. Platt, J. Chem. Phys., 17, 484 (1949).

14. A. Moscowitz, in "ORD-Applications to Organic Chemistry," C. Djerassi Ed., McGraw Hill, New York, N.Y., 1960, p 150.

15. P. Pino, F. Ciardelli, and E. Chiellini, in "Optical Rotatory Dispersion and Circular Dichroism in Organic Chemistry," G. Snatzke Ed., Heyden \& Sons Ltd., London, 1967, p 341.

16. C. Villiers, C. Braud, M. Vert, and E. Chiellini, unpublished results.

17. C. Villiers, C. Braud, M. Vert, E. Chiellini, and M. Marchetti, Eur. Polym. J., 14, 211 (1978).

18. T. Sato, T. Kadowaki, H. Takeda, and T. Otsu, $J$. Macromol. Sci., Chem., A12, 1225 (1978).

19. E. Kiesper, W. Gronski, and V. Barth, Makromol. Chem., 139, 1 (1970). 